\title{
Funktionsverbgefüge - empirisch (am Beispiel von kommen)*
}

\author{
Alain Kamber (Neuchâtel)
}

\begin{abstract}
In grammars and textbooks used to teach German as a foreign language, the chapters devoted to the "Funktionsverbgefüge" (light verb constructions) are based on data that are mainly obtained by introspection. German dictionaries also often seem not to be based on empirical data. Through the example of kommen (to come), the present paper aims at showing a few new and decisive elements that empirical, strictly corpus-driven research, by means of the new digital corpora, makes it possible to highlight. The purpose is to achieve a description that is more precise and more faithful to the target language in foreign languages teaching.
\end{abstract}

Keywords: Light verb construction - light verb - corpus linguistics - empirical research digital corpora - COSMAS II - prototype semantics - syntax - valence - complementation lexicology - grammar

\section{$1 \quad$ Einleitung}

$\mathrm{Zu}$ den Funktionsverbgefügen (FVG) im Deutschen gibt es seit den ersten Studien der "Gründerväter" von Polenz und Daniels aus dem Jahre 1963 inzwischen eine umfangreiche Literatur. Doch was in den zum Thema veröffentlichten Studien weiterhin fehlt, ist zweifelsohne eine Verankerung der Behauptungen in einer ausreichenden Datenbank. Oder wie es Van Pottelberge (2001:8) formuliert: "Eine umfassende Kritik, die nicht nur theoretisch, sondern auch empirisch vorgeht, fehlt ... bis auf den heutigen Tag." (Dies bleibt allerdings auch nach seiner eigenen Untersuchung zu den FVG der Fall).

Aufgrund dieses empirischen Defizits sind einige fundamentale Fragen bis heute nicht zufriedenstellend beantwortet worden, wie zum Beispiel: Welche sind die frequentesten FVG im alltäglichen Sprachgebrauch? Welche wichtigen FVG stehen (nicht) in einsprachigen Wörterbüchern des Deutschen? Welche wichtigen FVG stehen (nicht) in DaF-Grammatiken? usw.

\footnotetext{
* Dieser Beitrag ist ein Auszug aus Kamber, Alain (im Druck): Funktionsverbgefüge - empirisch. Eine korpusbasierte Untersuchung in fremdsprachendidaktischer Perspektive. Dissertation zur Erlangung der Doktorwürde an der Philosophischen Fakultät der Universität Neuchâtel.
} 


\section{$2 \quad$ Wahl der Funktionsverben}

Um diese Lücken zu schließen, mussten als erstes die Funktionsverben selbst, oder zumindest einige repräsentative Vertreter davon ausgewählt werden. Eine auf der Analyse von Wörterbüchern basierende unveröffentlichte Diplomarbeit von Anne Hofstetter (1989) zu den FVG vom Typ Zustands- oder Bewegungsverb + PG (mit abstraktem ODER konkretem Substantiv) hat gezeigt, welche Funktionsverben am "produktivsten" sind: ${ }^{1}$

\begin{tabular}{|l|l|l|}
\hline Verb & $\begin{array}{l}\text { Anzahl types } \\
\text { (in den } \\
\text { Wörterbüchern) }\end{array}$ & in \% \\
\hline 1. bringen & 279 & 15,74 \\
\hline 2. sein & 221 & 12,46 \\
\hline 3. kommen & 206 & 11,62 \\
\hline 4. sich befinden & 138 & 7,78 \\
\hline 5. stehen & 133 & 7,5 \\
\hline 6. geraten & 109 & 6,14 \\
\hline 7. nehmen & 61 & 3,44 \\
\hline 8. stellen & 59 & 3,3 \\
\hline 9. bleiben & 57 & 3,21 \\
\hline 10. setzen & 56 & 3,16 \\
\hline 11. gehen & 47 & 2,66 \\
\hline 12. halten & 46 & 2,6 \\
\hline 13. versetzen & 39 & 2,2 \\
\hline 14. haben & 35 & 1,97 \\
\hline 15. treten & 30 & 1,7 \\
\hline 16. gelangen & 23 & 1,3 \\
\hline 17. liegen & 22 & 1,24 \\
\hline 18. geben & 20 & 1,13 \\
\hline 19. begriffen sein & 19 & 1,07 \\
\hline 20. verfallen & 17 & 0,96 \\
\hline & & \\
\hline
\end{tabular}

\begin{tabular}{|l|l|l|}
\hline Verb & $\begin{array}{l}\text { Anzahl types } \\
\text { (in den } \\
\text { Wörterbüchern) }\end{array}$ & in \% \\
\hline 21. fallen & 17 & 0,96 \\
\hline 22. führen & 16 & 0,9 \\
\hline 23. ziehen & 15 & 0,85 \\
\hline 24. stürzen & 14 & 0,79 \\
\hline 25. lassen & 13 & 0,73 \\
\hline 26. ergehen & 13 & 0,73 \\
\hline 27. legen & 12 & 0,68 \\
\hline 28. ausbrechen & 10 & 0,56 \\
\hline 29. schreiten & 9 & 0,51 \\
\hline 30. treiben & 8 & 0,45 \\
\hline 31. stoßen & 7 & 0,34 \\
\hline 32. schweben & 5 & 0,28 \\
\hline 33. versinken & 4 & 0,23 \\
\hline 34. sinken & 3 & 0,17 \\
\hline 35. stecken & 2 & 0,11 \\
\hline 36. schlagen & 2 & 0,11 \\
\hline 37. jagen & 2 & 0,11 \\
\hline 38. fassen & 2 & 0,11 \\
\hline 39. werfen & 1 & 0,06 \\
\hline 40. tragen & 1 & 0,06 \\
\hline Total & $\mathbf{1} \mathbf{7 7 3}$ & $\mathbf{2 1 0 0}$ \\
\hline & & \\
\hline & 13 & \\
\hline
\end{tabular}

Tab. 1: "Produktivität" der Funktionsverben in FVG vom Typ Zustands- oder Bewegungsverb + PG (mit abstraktem oder konkretem Substantiv) ${ }^{2}$

Im Rahmen meiner empirischen Untersuchung habe ich beschlossen, mich auf die 10 "produktivsten" Funktionsverben zu konzentrieren, wobei ich aus praktischen Gründen auf das Funktionsverb sein verzichtet habe. Die in meiner Arbeit besprochenen Verben sind daher: bringen, kommen, sich befinden, stehen, geraten, nehmen, stellen, bleiben, setzen und gehen.

\footnotetext{
${ }^{1}$ Diese Liste würde bloß geringfügige Abweichungen aufweisen, wenn man von der Untersuchung von ManSeob So (1991) ausgehen würde.

2 Tabelle nach Hofstetter (1989: 60).
} 
Im Rahmen dieses Beitrags sollen einige Ergebnisse zum Funktionsverb kommen vorgestellt werden.

\section{Ausgewählte Datenbank: die Spiegel-CD-Rom}

Das Ziel der vorliegenden Arbeit ist, ein möglichst genaues und vollständiges Bild der Stellung von FVG in der deutschen Standardsprache der Gegenwart zu vermitteln. In diesem Zusammenhang schien mir das Nachrichtenmagazin Der Spiegel für meine Untersuchungen eine geeignete Grundlage zu bieten. Man kann die in der Zeitschrift abgedruckten Texte wohl als einigermaßen repräsentativ für die aktuelle journalistische Sprache in Berichterstattungen betrachten.

Die CD-Rom enthält die 52 im Jahre 1997 erschienenen Nummern des Spiegel, was für mein Unterfangen einen angemessenen Umfang repräsentiert. Das Korpus bietet unter anderem die Möglichkeit, absolute Zahlen zu eruieren.

Im Sprachreport 2/2000 schreibt Ulrike Haß-Zumkehr über digitale Textkorpora:

"Um solche Korpora aber für lexikologische und lexikografische Zwecke nutzen zu können, sind eine Reihe teils linguistischer, teils informationeller Arbeiten nötig. Texte müssen möglichst in digitaler Form beschafft, danach in ein geeignetes Datenformat konvertiert, von "unbrauchbaren" Kodierungen (z.B. Formatierungsinformationen) gereinigt und anschließend mit besonderen Kodierungen versehen werden, damit wissenschaftliche Nutzer etwa Überschriften und Absätze identifizieren können; hinzu kommt die bibliografische Dokumentation einschließlich Angaben zur Entstehungszeit, zur Textsorte u.ä., die für den automatisch generierten Quellennachweis der Belege, aber auch für die Zusammenstellung spezifischer Ad-hoc-Korpora durch die Nutzer selbst benötigt werden." (Haß-Zumkehr 2000: 5)

Von diesen idealen Voraussetzungen ist die Spiegel-CD-Rom weit entfernt. Sie ist nämlich keineswegs auf sprachwissenschaftliche Bedürfnisse zugeschnitten, sondern wurde vielmehr für journalistische, zeitgeschichtliche oder sogar soziologische Forschungen konzipiert. Kurz: Sie enthält "unpräpariertes" Material in PDF-Format; das Korpus wurde keiner "Voranalyse" unterzogen und es ist nicht linguistisch annotiert ("getaggt").

Was eine solche Software vor allem vermissen lässt, ist ein Lemmatisierer, ein morphologisches Analyseprogramm, durch das die unterschiedlichen Wortformen, die in den Texten vorkommen (z.B. komme, kam, kommen, gekommen usw.), einer Grundform (kommen) zugeordnet werden.

So bleibt einem nichts anderes übrig, als sich durch die 12'472 Seiten der CD-Rom durchzukämpfen. Dies geschieht in vier Schritten:

1. Eingabe des Suchbegriffs. Jede Verbform wird als Suchbegriff eingegeben, um ein vollständiges Bild zu bekommen (für das Präsens: komme, kommst, kommt usw.).

2. Ausscheiden von irrelevanten Verbformen. Bestimmte "Schlacken" werden beseitigt, wie substantivierte Infinitive (das Kommen, sein Kommen), Substantive, Adjektive oder Adverbien, die den Suchbegriff zwar enthalten, jedoch keinen Platz im zu schaffenden Korpus haben (Nach/kommen bzw. voll/kommen) oder auch Verben mit Präfix oder 
Verbzusatz (beispielsweise be/kommen; ent/kommen bzw. abkommen, ankommen, aufkommen, auskommen, beikommen usw.) Das Korpus enthält also auschließlich einfache Verbformen.

3. Disambiguierung von Homonymen. Die verschiedenen Verwendungen der einzelnen Verbformen müssen unterschieden werden. Kommen zum Beispiel kann für 8 verschiedene finite Formen stehen: erste und dritte Person Plural sowie Höflichkeitsform im Indikativ Präsens (wir kommen; sie kommen; Sie kommen), dieselben Kategorienkombinationen im Konjunktiv I, erste Person Plural und Höflichkeitsform im Imperativ. Dazu kommt als neunte Verwendung der Infinitiv.

4. Bestimmung des Verbgebrauchs. Jeder Beleg von kommen muss darauf hin untersucht werden, ob das Verb als Funktionsverb fungiert oder nicht.

Diese Prozedur ist zwar langwierig und mühsam, doch sie birgt auch Positives: Sie zwingt einen, nichts vorwegzunehmen (was beispielsweise bei einer Booleschen Suche zwangsläufig der Fall wäre) und, vom Verb ausgehend, eine genaue und exhaustive Beschreibung des Phänomens zu erstellen. Denn eines ist klar: Bei einer streng empirischen Untersuchung gilt das Arbeitsprinzip: First throw away your evidence! (vgl. Sinclair 1986).

\section{Die Prototypensemantik als Instrument der Analyse}

Es geht mir hier keineswegs um einen erneuten Versuch, vermeintliche allgemeingültige Definitionen und verbindliche Abgrenzungskriterien für FVG aufzustellen. Die Forschung hat sich in dieser Hinsicht wohl wirklich in eine Sackgasse hineinmanövriert, wie Van Pottelberge (2001) in seiner Einleitung feststellt. Genauso wenig geht es mir aber darum, den Begriff selbst als letztendlich irrelevant zu verwerfen. Vielmehr soll der Versuch unternommen werden, von der Sprachrealität ausgehend eine Sammlung von Syntagmen zusammenzustellen, die gemeinsame semantische und syntaktische Merkmale aufweisen.

Um dieses Unterfangen in Angriff zu nehmen, braucht es einen möglichst flexiblen theoretischen Rahmen. Die verschiedenen Versuche, um diese Konstruktionen abzugrenzen, sind wohl vor allem daran gescheitert, dass sie Definitionskriterien im Sinne der klassischen Logik aristotelischer Prägung aufstellen wollten. Definitionsversuche anhand von notwendigen und hinreichenden Bedingungen stoßen aufgrund der Heterogenität der Kriterien (Semantik, Syntax, Etymologie) bald an Grenzen, so dass die Autoren gleich im Anschluss an die eigene Definition hinzufügen müssen (wie beispielsweise Hentschel/Weydt 1990: 79), dass die von ihnen aufgeführten Kriterien (genannt werden die Ersetzbarkeit durch ein einfaches Verb, die fehlende Pronominalisierbarkeit, der festgelegte Artikelgebrauch usw.) "indessen regelmäßig nicht für alle Mitglieder dieser Gruppe zutreffen". Oder aber sie nehmen gleich Vertreter der Kategorie in ihre Liste auf, welche die eigenen Kriterien nicht erfüllen, wie beispielsweise Helbig/Buscha (2001: 87): Obwohl als Kriterium "Verbal- bzw. Adjektivabstraktum" postuliert wird, enthält deren Liste u. a. die FVG Angst haben, zur Vernunft kommen und in Zorn geraten. 
Als Rahmen schlage ich deshalb das Modell der umrahmten Schnittmengen vor. Trotz aller Unterschiede weisen die Syntagmen, die der Kategorie FVG zugesprochen werden, alle eine Konstante auf: Sie bestehen aus einem Funktionsverb (mit "abgeschwächter" Bedeutung) und aus einem nicht-verbalen Teil (Nominalgruppe im Akkusativ oder Präpositionalgruppe), der die Hauptbedeutung des gesamten Ausdrucks trägt.

Der "Bedeutungsverlust" des Verbs ist m. E. das grundlegende Kriterium [hier Kriterium A], da ein Verb keineswegs per se Funktionsverb ist. ${ }^{3}$ Neben dem Basis-Kriterium [A], das allen Mitgliedern der Kategorie gemeinsam ist, können in diesem Repräsentationsmodell nun weitere Kriterien berücksichtigt werden, welche nicht immer erfüllt sind. Die Anzahl von Definitionskriterien innerhalb des Rahmens ist im Prinzip nicht begrenzt. Unter den verschiedenen Möglichkeiten der Kategorisierung habe ich drei herangezogen:

$1^{\circ} \quad$ Ist das Substantiv ein Verbalabstraktum oder nicht? [Kriterium B]

$2^{\circ} \quad$ Ist das Verb ein Bewegungs- bzw. Zustandsverb oder nicht? (nehmen, geben, bringen, kommen, setzen, stellen, stehen usw.)? [Kriterium C]

$3^{\circ} \quad$ Enthält das Syntagma eine Präpositionalgruppe oder nicht? [Kriterium D]

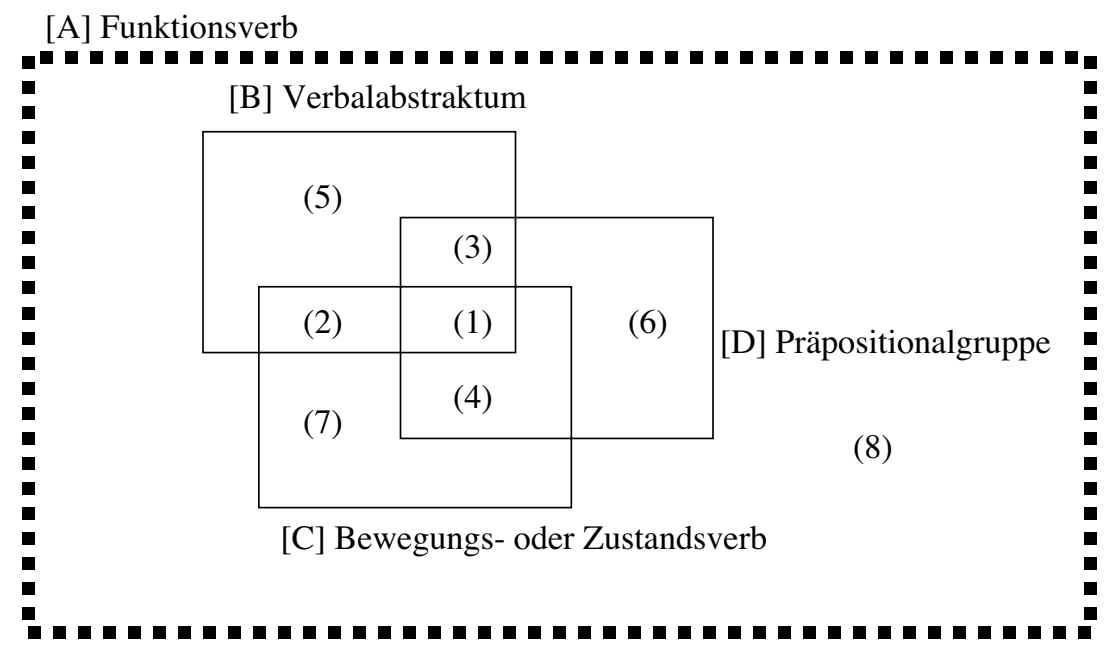

(9)

Schema 1: Subklassifizierung der FVG im Modell der umrahmten Schnittmengen ${ }^{4}$

Diese Kombination von grundsätzlich verschiedenen Kriterien (Semantik, Morphologie, und sogar Syntax) ist natürlich kritisierbar, doch das ist m. E. die einzige Möglichkeit, ein komplexes Phänomen einzugrenzen und ihm letztendlich gerecht zu werden. Die vier oben aufgeführten Kriterien erlauben es, 9 Fälle zu unterscheiden:
(1) zum Ausdruck kommen
(2) Stellung nehmen
(3) in Besitz haben
(4) zur Welt kommen
(5) Ordnung schaffen

\footnotetext{
3 Oder wie Vivès (1983: 8) es formuliert: "un verbe n'est pas support par définition".

${ }^{4}$ Zum Einsatz der Prototypensemantik zur Klassifizierung von FVG und zum Modell der umrahmten Schnittmengen vgl. Kamber/Näf (2001: 905ff.).
} 
(6) in Angst halten

(7) Platz nehmen

(8) Lust haben/Licht machen

(9) zur Geburtstagsfeier gehen/Zucker nehmen/Kuchen essen

Unter den obigen Syntagmen ist das Exemplar (1) ein prototypisches FVG, denn es erfüllt die 4 festgelegten Kriterien: Funktionsverb (mit "verblasster" Bedeutung) [A]; Verbalabstraktum [B]; Bewegungsverb [C]; Präpositionalgruppe [D]. Die Nummern (2), (3) und (4) nehmen eine etwas weniger zentrale Stelle ein, da (2) keine Präpositionalgruppe, (3) kein Bewegungsoder Zustandsverb und (4) kein Verbalabstraktum enthält. Die Beispiele (5), (6) und (7) befinden sich noch etwas weiter an der Peripherie, da sie nur noch eines der Kriterien [B], [C] oder [D] erfüllen, während (8) zwar keines davon erfüllt, jedoch trotzdem als FVG zu betrachten ist, da es die Grundbedingung [A] erfüllt. (9) schließlich steht außerhalb des Rahmens und ist kein FVG.

\section{$5 \quad$ kommen als Funktionsverb}

Für das Verb kommen liefert das Spiegel-Korpus ingesamt 6'441 Belege. Unter den im Rahmen meiner Studie untersuchten Verben ist kommen somit das frequenteste.

Von den 6'441 Verbformen kommen bloß 1'549, d. h. nur 24\% in FVG vor. Das ist nach bleiben $(6,3 \%)$ und gehen $(16,6 \%)$ der drittniedrigste Anteil.5 Dieses deutliche Ergebnis bedeutet schlicht, dass kommen in der deutschen Gegenwartssprache in erster Linie als Vollverb fungiert.

Die 1'549 Belege verteilen sich auf 214 Substantive, wobei 2 Substantive in 2 verschiedenen FVG auftauchen: Hand (abhanden kommen und in js/<bestimmte $>$ Hände kommen) und Recht (zurecht kommen und zu seinem Recht kommen), so dass wir insgesamt auf 216 types kommen. Das Funktionsverb kommt - wie ansonsten nur geraten, sich befinden und bleiben ausschließlich in Verbindung mit einer Präpositionalgruppe vor.

\subsection{Allgemeines zu den Funktionsverbgefügen mit kommen}

Die Zahl von 216 verschiedenen FVG mit kommen mag auf den ersten Blick recht beeindruckend sein. Doch es ist möglich, nach semantischen Kriterien zusammenhängende Gruppen oder Typen zu eruieren und dadurch diese Zahl zu "verringern". Dabei begegnen uns in den PG (Quasi-)Synonyme, wie beispielsweise Idee/Einfall oder Lage/Situation.

Auch stilistische Varianten kommen vor. Ein schönes Beispiel dafür liefern die 3 Substantive Gefängnis, Knast und Vollzug (unmarkiert, umgangssprachlich bzw. amtsdeutsch); neben der allgemeinen Bezeichnung Gefängnis finden wir auch noch die spezifische russische Ausführung des Strafvollzugs: den Gulag.

\footnotetext{
5 geraten: $71,5 \%$; stellen: $68,7 \%$; bringen: $56,4 \%$; nehmen: $51,5 \%$; sich befinden: $50,1 \%$; setzen: 45,3\%; stehen: $39,8 \%$; gehen: $16,6 \%$; bleiben: $6,3 \%$.
} 
Schließlich sind im Korpus auch (Teil)Synonyme vorhanden, die unterschiedliche Realitäten beschreiben: Neben dem allgemeinen Substantiv Liste steht der Index, das Verzeichnis der für Katholiken verbotenen Bücher (Index [Librorum Prohibitorum]).

Die quantitativ bedeutendste Gruppe ist jedoch nicht nach einem semantischen Kriterium zusammengestellt, sondern nach einem syntaktischen: 19 FVG teilen als gemeinsames Merkmal den substantivierten Infinitiv.

Die unten aufgeführten Kategorien sind nicht als völlig undurchlässig anzusehen. (Die FVG werden gekürzt zitiert; in Klammern die Anzahl von Belegen):

- ums Leben kommen (79) - Syntagmen mit der Bedeutung '(plötzlich) sterben'. Ferner: zu Tode kommen (10).

- zustande kommen (71) - Syntagmen mit der allgemeinen Bedeutung 'bewerkstelligt werden'. Ferner: auf die Beine kommen (7).

- auf eine <best.> Idee kommen (59) - Syntagmen mit der Bedeutung 'eine Idee, einen Gedanken, einen Einfall haben'. Ferner: auf einen <best.> Einfall kommen (1); auf einen <best.> Gedanken kommen (9); auf ein Konzept kommen (1).

- $\quad$ zu einem < best.> Ergebnis kommen (59) - Syntagmen mit terminativer Bedeutung '(nach längerer Überlegung oder Arbeit) ein Endergebnis erzielen'. Ferner: zu einer $<$ best.> Abschätzung kommen (1); zu einer <best.> Auffassung kommen (1); zu einer $<$ best.> Auslegung kommen (1); zu einer <best.> Beurteilung kommen (1); zu einer $<$ best.> Bilanz kommen (1); zu einer Diagnose kommen (1); zu einer <best.> Einschätzung kommen (2); zu <einer best.> Einsicht kommen (3); zu einer <best.> Entscheidung kommen (2); zu <einer best.> Erkenntnis kommen (9); zu <einem best.> Fazit kommen (3); zu <einer best.> Meinung kommen (1); zu einem <best.> Resultat kommen (4); zu einem <best.> Schluss kommen (43); zu <einer best.> Schlussfolgerung kommen (3); zu <einer best.> Überzeugung kommen (7).

- auf den Markt kommen (54) - Syntagmen mit der Bedeutung 'produziert werden und zum Verkauf angeboten werden'. Ferner: in den Handel kommen (5).

- in Gang kommen (32) - Syntagmen mit inchoativer Bedeutung 'initiiert werden; beginnen'. Ferner: in Bewegung kommen (1); in Fahrt kommen (6); in Schwung kommen (6); auf Touren kommen (7); auf den Weg kommen (2).

- zum Einsatz kommen (28) - Syntagmen mit der Bedeutung 'verwendet werden; aktiv werden'. Ferner: zum Zug(e) kommen (23).

- ans Licht kommen (27) - Syntagmen mit der Bedeutung 'bekanntgemacht werden; preisgegeben werden'. Ferner: an die Öffentlichkeit kommen (1); zutage kommen (8); zum Vorschein kommen (16).

- jm/etw. auf die Spur kommen (27) - Syntagmen mit der Bedeutung 'jn entlarven; etw. enthüllen'. Ferner: jm auf die Schliche kommen (9). 
- ins Gefängnis kommen (15) - Syntagmen mit der Bedeutung 'verhaftet werden; eingesperrt werden'. Ferner: in Gefangenschaft kommen (1); in den Gulag kommen (1); in Haft kommen (11); in den Knast kommen (3); in den Vollzug kommen (1).

- zur Sprache kommen (15) - Syntagmen mit der Bedeutung 'explizit ausgedrückt werden; gesagt werden'. Ferner: zum Ausdruck kommen (7).

- in Berïhrung kommen (9) - Syntagmen mit der Bedeutung 'mit jm/etw. zum ersten Mal konfrontiert werden'. Ferner: in Kontakt kommen (4); in Verbindung kommen (1).

- auf <eine best.> Liste kommen (8) - Syntagmen mit der Bedeutung '(unfreiwillig) die öffentliche Aufmerksamkeit auf sich ziehen'. Ferner: auf die Frontseite kommen (1); auf den Index kommen (3); in <ein best.> Register kommen (1); in die Schlagzeilen kommen (2); in die Zeitung kommen (2).

- in die Bredouille kommen (6) - Syntagmen mit der Bedeutung 'ernsthafte Probleme (oft finanzieller Art) bekommen'. Ferner: in Bedrängnis kommen (3); unter Druck kommen (4); auf den Hund kommen (1); in Not kommen (2); in Schwierigkeiten kommen (4); in <einen best.> Strudel kommen (1); in Turbulenzen kommen (2).

- in eine <best.> Lage kommen (5) - Syntagmen mit ähnlicher, jedoch "neutralerer" Bedeutung 'einer neuen (nicht zwangsläufig problematischen) Situation begegnen'. Ferner: in <eine best.> Situation kommen (1).

- in Form kommen (3) - Syntagmen mit der Bedeutung '(wieder) den gewünschten Zustand erlangen'. Ferner: auf <ein best.> Niveau kommen (1); in Ordnung kommen (1); auf Trab kommen (1).

- aus dem Gleichgewicht kommen (1) - Syntagmen mit der Bedeutung 'sich (unerwartet) der Kontrolle entziehen; den normalen, geordneten Weg verlassen'. Ferner: aus dem Tritt kommen (1).

- unter die Leute kommen (1) - Syntagmen mit der Bedeutung '(für eine Ware:) verkauft werden; verbreitet werden'. Ferner: an den Mann kommen (1).

- in <einen best.> Verdacht kommen (1) - Syntagmen mit der Bedeutung '(als Person; durch sein Tun) in Frage gestellt werden'. Ferner: in den Geruch <von etw.> kommen (1); in Verruf kommen (1); in Verlegenheit kommen (1).

- Belege mit subtantiviertem Infinitiv. Typ: ins Plaudern kommen (3) - Syntagmen mit der inchoativen Bedeutung '(unfreiwillig) mit einer Tätigkeit beginnen'. Ferner: ins Drucksen kommen (1); zum Erliegen kommen (2); ins Grübeln kommen (2); zum Halten kommen (1); zum Nachdenken kommen (1); ins Philosophieren kommen (1); ins Reden kommen (1); ins Rotieren kommen (1); ins Rutschen kommen (3); ins Schlingern kommen (1); ins Schwanken kommen (1); ins Schwitzen kommen (1); zum Stehen kommen (2); ins Stocken kommen (3); ins Stolpern kommen (1); zum Tragen kommen (3); ins Träumen kommen (1); ins Wanken kommen (2). 
Einige wenige Bemerkungen zu dieser Liste:

Unter den 20 Gruppen zählt neben derjenigen mit substantiviertem Infinitiv in der PG und inchoativer Bedeutung (19x) diejenige von zu einem <best.> Ergebnis kommen (17x) die meisten types.

Die dominierende Stellung der FVG mit substantiviertem Infinitiv ist jedoch in Bezug auf die types (19 von 216, d. h. 8,8\%) und noch vielmehr in Bezug auf die tokens (32 von 1549 Belegen, 2 Prozent) keineswegs so ausgeprägt wie bei bringen. ${ }^{6}$ Obwohl das Muster recht produktiv ist, nehmen diese Syntagmen zahlenmäßig nur einen geradezu unbedeutenden Platz ein: Nur gerade 4 von ihnen kommen im Korpus 3 mal vor.

Auffallend ist innerhalb dieser Gruppe die Vorherrschaft der zusammengezogenen Präposition ins (14x), die wohl den "unfreiwilligen Charakter" der Handlung unterstreicht, im Vergleich zu zum (5x). Diese Hypothese wird dadurch gestützt, dass die Syntagmen mit geraten und substantiviertem Infinitiv ausschließlich diese Präposition enthalten.

Die 5 types mit zum scheinen außerdem eine leicht andere Bedeutung als diejenigen mit ins zu haben:

Im Fall von Nachdenken zum Beispiel ist die Handlung wohl recht positiv konnotiert: Das kritische Hinterfragen von Informationen kann zwar von Drittpersonen - wie in [1] - als ungemütlich oder gar unangenehm empfunden werden, doch es ist zweifellos ein bewusster Willensakt:

(1) Das war so imposant vorgetragen, dass niemand im Publikum zum Hinterherdenken, geschweige denn zum Nachdenken gekommen ist.

Demgegenüber steht bei den mit ins angeschlossenen Infinitiven ein passives Hineinschliddern in ein planlos-kreisendes, bei Grübeln geradezu quälendes und ergebnisloses Sinnieren im Vordergrund.

(2) Da räkelt sich Manfred Krug in der TV-Rolle des Anwalts Liebling bei der Geliebten auf dem Sofa und kommt ganz entspannt im Hier und Jetzt ins Philosophieren über die Gebresten des Alters [...].

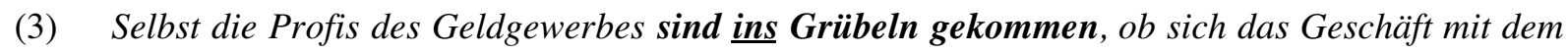
Geld nicht allzuweit von der ökonomischen Realität entfernt hat.

In der zweitgrößten Gruppe um das FVG zu einem <best.> Ergebnis kommen fällt auf, dass das deutsche Substantiv Ergebnis (59x) dem synonymischen Lehnwort Resultat (4x) eindeutig vorgezogen wird (auch Schluss, Erkenntnis und Überzeugung sind häufiger vertreten). Unter semantischem Gesichtspunkt ist jedoch kein Unterschied feststellbar und in den Belegen (4) und (5) ließe sich Resultat jeweils durch Ergebnis ersetzen:

(4) Die New Yorker Therapeutin Yvette Colón berichtet jetzt in der "Village Voice" über Erfahrungen bei der computergestützten Gruppentherapie und kommt zu durchaus positiven Resultaten.

\footnotetext{
658 von 350 oder ein Sechstel der types; 8,4\% der tokens (131 von 1'553).
} 
(5) Wir fassen nur alle Möglichkeiten ins Auge, um bei den Verhandlungen über die Rückgabe der jüdischen Guthaben am 10. Februar in New York endlich zu einem Resultat zu kommen.

Im Vergleich zu deren Pendant bei bringen erweist sich die Gruppe um unter die Leute kommen (drittletzte Gruppe) als wenig entwickelt: Während die Kategorie bei bringen mit nicht weniger als 7 verschiedenen Substantiven (Volk, Frau, Käufer, Kunde, Kundschaft, Leute, Mann) recht vielseitig und produktiv ist, bietet das Korpus als einzige Variante zu unter die Leute kommen das Syntagma an den Mann kommen:

(6) Die EU-Staaten beschließen mit Hilfe spitzfindiger Vertragsauslegungen, das Projekt auf ein späteres Datum zu verschieben - die Euro-Scheine kämen beispielsweise erst 2005 unter die Leute.

(7) Ich werde Ihnen jetzt mal ein Marketing verschreiben, damit Ihr Produkt wirklich an den Mann kommt.

Auch unter dem Aspekt der tokens ist diese Kategorie bei kommen geradezu bedeutungslos: Beide Syntagmen sind bloß einmal belegt. Hier - wie auch anderswo - lässt sich beobachten, dass die in einigen Handbüchern unterstellte Parallelität zwischen bringen und kommen sich bei einer empirischen Überprüfung weitgehend als Illusion erweist.

Weiter fungiert das Funktionsverb kommen in einigen FVG als Variante von geraten. Dies trifft beispielsweise auf in Verdacht kommen [1x, vgl. 8] und in Verruf kommen [1x, vgl. 9] $\mathrm{zu}:$

(8) Anthony-Noel Kelly, 41, britischer Bildhauer, kam in den Verdacht der Leichenfledderei zu künstlerischen Zwecken.

(9) Vor allem die besonders genügsamen Diesel-Direkteinspritzer modernster Bauart kommen durch die amerikanischen Forschungsergebnisse zunehmend in Verruf.

Diese Syntagmen erfreuen sich jedoch einer weit geringeren Beliebtheit als diejenigen mit geraten (in/unter Verdacht geraten: 29x; in Verruf geraten: 10x), obwohl letzteres Verb im gesamten Korpus wesentlich weniger häufig vorkommt als kommen. Eine Situation, die sich dadurch erklären lässt, dass geraten "ausdrucksvoller" ist, dass der unfreiwillige Charakter der Handlung, die wehrlose Haltung des (meist menschlichen) Subjekts bei diesem Funktionsverb deutlicher unterstrichen wird.

Dieser Befund trifft auch auf das Syntagma in Verlegenheit kommen zu (1x), welches dieselbe Bedeutung hat wie in Verlegenheit geraten (2x):

(10) Wir sollten in einem freien Land leben, als ob über uns irgendwo Buch geführt würde, auf dass wir nicht eines Tages angesichts einer Akte in Verlegenheit kämen. 


\subsection{Die 10 häufigsten Funktionsverbgefüge mit kommen}

Ich komme nun zum "Herzstück" meiner Arbeit: Die folgende Tabelle zeigt, welche Substantive am häufigsten in Verbindung mit dem Funktionsverb kommen auftreten:

\begin{tabular}{|c|c|c|c|}
\hline & Substantiv & Anzahl Belege & FVG \\
\hline 1. & Leben & 79 & ums Leben kommen \\
\hline 2. & Stand (in: zustande) & 71 & zustande kommen \\
\hline 3. & Welt & 62 & zur/auf die Welt kommen \\
\hline 4. & Frage & 61 & (für jn/etw.) in Frage kommen \\
\hline 5. & Idee & 60 & auf eine $<$ bestimmte $>$ Idee kommen \\
\hline 6. & Ergebnis & 59 & $\mathrm{zu}$ einem $<$ bestimmten $>$ Ergebnis kommen \\
\hline 7. & Markt & 54 & auf den Markt kommen \\
\hline 8. & Gut (in: zugute) & 51 & jm zugute kommen \\
\hline 9. & Hand (in: abhanden) & $\begin{array}{l}(48) \\
(3)\end{array}$ & $\begin{array}{l}\text { (jm) abhanden kommen } \\
\text { in js/<bestimmte }>\text { Hände kommen }\end{array}$ \\
\hline 10. & Schluss & 43 & zu einem $<$ bestimmten $>$ Schluss kommen \\
\hline
\end{tabular}

Tab. 2: Top-Ten: Die häufigsten Substantive im Zusammenhang mit kommen in FVG

Die 10 häufigsten Substantive, die mit dem Funktionsverb kommen kombiniert sind, machen zusammen 591 Belege, d. h. 38,2\% aller FVG mit diesem Verb aus. Wir haben also eine Situation mit einer bedeutenden Anzahl von Substantiven in FVG und einer großen Streuung der Verbformen über die Substantive. ${ }^{7}$

Bei der Besprechung der einzelnen Substantive sollen die folgenden Gesichtspunkte zur Sprache kommen:

- Erweiterbarkeit der PG bzw. NG: Artikelgebrauch; evt. andere auftretende Erweiterungen (Adjektiv, Vorderglied in Kompositum, Relativsatz, Genitivattribut, Präpositionalattribut usw.); Negation

- Komplementierung des FVG

- Syntaktische Aspekte (Satzgliedwert)

- Semantische Aspekte (semantische Restriktionen bei den Komplementen ([+Hum], [+Anim], [+Abstr], [+Konkr], [+Koll]); häufige Substantive (event. "Archilexeme")

- Sonstiges (z.B. Klammerbildung)

Im Folgenden sollen bloß die 4 frequentesten Syntagmen mit kommen vorgestellt und erläutert werden. Für die restlichen Syntagmen vgl. Kamber (im Druck):

\footnotetext{
7 Zum Vergleich: stellen: 66\% aller FVG mit diesem Verb; bleiben: 51,4\%; setzen: 50,1\%; nehmen: 46\%; stehen: 40,9\%; sich befinden: 39,7\%; gehen: $35,3 \%$; geraten: $35 \%$; bringen: $20,3 \%$.
} 


\section{I. ums Leben kommen}

(sterben; sein Leben verlieren; den Tod finden)

Das FVG ums Leben kommen ist mit 79 Belegen das am häufigsten vorkommende Syntagma mit dem Funktionsverb kommen.

Zur Erweiterbarkeit. In der PG sind Präposition und Definitartikel fest und treten stets zusammengezogen auf. Auffallend ist im Zusammenhang mit diesem Syntagma die - wohl vor allem durch die Textsorte zu erklärende - hohe Anzahl von Vergangenheitstempora (48x Präteritum; 21x Partizip II; 3x Partizipialattribute). Das Syntagma wird nie erweitert, wie in folgenden Belegen:

(11) Ein Passagier kam ums Leben.

(12) Der Geistliche kann immer noch nicht fassen, dass außer seinen Peinigern nur eine Geisel und zwei Soldaten ums Leben kamen.

(13) Und mit derselben Nonchalance, mit der die Stasi sein Äußeres beschreibt ("Seine Kleidung befindet sich stets in einem guten Zustand"), wird auch seine Familiengeschichte evaluiert: "Seine Eltern sollen während der Nazi-Zeit ums Leben gekommen sein (vergast)."

Zur Komplementierung. Das Syntagma lässt keine Komplementierung im Sinne eines Akkusativ-, Dativ- oder Präpositionalobjekts zu.

Subjekt. "Ums Leben" kommen ausschließlich menschliche Wesen. Andere semantische Kategorien sind an der Subjektstelle nicht vertreten.

Adverbial. In nicht weniger als 51 Belegen werden die Ursache oder die Umstände für den meist jähen, manchmal sogar gewaltsamen Tod - des Subjekts angeführt. Dies geschieht 44mal mittels einer durch bei eingeleiteten Präpositionalgruppe; in 6 weiteren Fällen wird diese durch durch eingeleitet; in einem Beleg schließlich steht im Verlauf an dieser Stelle.

56\% aller Belege enthalten eine Präpositionalgruppe mit bei, welche die Umstände für das plötzliche Ableben des Subjekts nennt. Unsere Belege liefern diesbezüglich ein recht genaues Spiegelbild unserer durch Gewaltanwendung geprägten Gesellschaft oder vielleicht bloß der auf Katastrophen fixierten Medienlandschaft. Als häufigste Todesursache gelten '(Verkehrs)unfälle' (11x Unfall; 7x Absturz; 3x Unglück) wie in [14; 15], 'Terroranschläge' (4x Anschlag; 2x Brand; 1x Attentat) wie in [16; 17], 'Militäreinsätze' (2x Kampfhandlungen; 1x Einsatz; 1x Manöver; 1x Kommandounternehmen; 1x Überfall) wie in [18] und 'sonstige Gewaltausbrüche' (4x Schießerei; 1x Schlägerei; 1x Demonstration) wie in [19]. Erst weit abgeschlagen treten 'Naturkatastrophen' (2x Erdbeben) auf.

(14) Bei einem Autounfall kommt der Maler ums Leben.

(15) Als er 1996 in Kroatien bei einem Flugzeugabsturz ums Leben kam, war er als USHandelsminister unterwegs.

(16) Der Attentäter kam bei dem Anschlag selbst ums Leben.

(17) Dreieinhalb Stunden später ist Amoussou tot - einer von zehn Menschen, die beim Brand der Asylbewerberunterkunft in der Lübecker Hafenstraße ums Leben kamen. 
(18) Ob die Mutter bei Kampfhandlungen ums Leben kam oder, wie einige Historiker behaupten, in deutscher Gefangenschaft unter Folter Verrat beging und erschossen wurde, ist nicht geklärt.

(19) Bei einer Schießerei unter Halbwüchsigen kam der 19jährige Kai-Uwe Gärtner ums Leben.

In 6 weiteren Belegen wird die Präpositionalgruppe mit durch eingeleitet. Dabei hat die PG entweder eine identische Bedeutung wie mit bei [vgl. 20] oder aber sie drückt die unmittelbare Todesursache, das todbringende Element selbst aus [vgl. 21]:

(20) Durch einen Brandanschlag in Krefeld waren drei Mitglieder einer seit langem in Deutschland lebenden türkischen Familie ums Leben gekommen, zwei Mädchen überlebten schwer verletzt.

(21) Durch Kugeln aus den Waffen der New Yorker Polizei sind in den letzten fünf Jahren mehr als hundert Bürger ums Leben gekommen, überwiegend Schwarze und Latinos.

Das Korpus liefert außerdem einen Beleg, in dem beide Formen von Adverbial vorhanden sind. In diesem Fall kommen die in [20] und [21] festgestellten, leicht unterschiedlichen semantischen Funktionen der Präpositionalgruppen (Umstände vs. Ursache/Verursacher) recht deutlich zur Geltung:

(22) Irgendwann in der Nacht könnten wir ums Leben kommen, bei einem Verkehrsunfall oder durch Messerstecher in der Dunkelheit.

Schließlich wird in einem Beleg das Satzglied durch im Verlauf eingeleitet. Die Bedeutung ist im wesentlichen dieselbe wie mit bei (Hunderte seiner Gegner kamen bei den missratenen CIA-Operationen ums Leben), doch wird dabei die zeitliche Ausdehnung betont:

(23) Nicht der Diktator in Bagdad, sondern Hunderte seiner Gegner kamen im Verlauf der mißratenen CIA-Operationen ums Leben.

Es sei an dieser Stelle angemerkt, dass 10 weitere Belege Ergänzungen enthalten, die zwar eine ähnliche semantische Funktion übernehmen, allerdings eher die Art und Weise des Ablebens als die Umstände bzw. die Ursache des Todes näher beschreiben oder erläutern (statt: Woran bzw. unter welchen Umständen ist er gestorben? antworten sie auf die Frage: Auf welche Art und Weise bzw. wie genau ist er gestorben?).

(24) Einige kamen auf nie geklärte Art und Weise ums Leben, andere verschwanden im Gefängnis.

(25) Die Internet-Mär will es, dass der Darwin-Preis alljährlich postum dem Unglücklichen verliehen wird, der auf die dümmste Art ums Leben kam.

(26) Als das mißlang, soll Kennedy die unbequeme Geliebte mit mehr als einer Million Dollar zum Schweigen bewegt haben, bis die Unglückliche von eigener Hand ums Leben kam.

\section{II. zustande kommen}

(verwirklicht, erreicht werden)

Auch beim FVG zustande kommen (71 Belege) ist der Artikelgebrauch strikte geregelt: Die Präposition $z u$ - mit dem Nullartikel - und das Substantiv Stand - stets mit der DativMarkierung $-e-$ kommen in unserem Korpus ausnahmslos zusammen- und kleingeschrieben vor. Die PG lässt keine Erweiterung zu. Typische Belege sehen folgendermaßen aus: 
(27) Ich bin bereit, Wetten in nahezu unbegrenzter Höhe abzuschließen, dass die Steuerreform in den nächsten Jahren zustande kommen wird.

(28) Sind die Entscheidungen zustande gekommen, legt sich die Aufregung.

(29) Tymar, so heißt es in dem Vertrag, [...] könne sich "dafür einsetzen, dass die von der Firma gewünschten und jeweils im Einzelfall vorher zu vereinbarenden Geschäfte zustande kommen".

(30) Die überparteiliche Mehrheit könnte zustande kommen, weil die CDU/CSU-Fraktion wahrscheinlich die Abstimmung in ihren Reihen freigeben wird.

(31) Von deren Lösung hänge es ab, ob ein Treffen der "großen Vier" zustande kommen würde.

(32) "Die Generalunternehmer akzeptieren Angebote, von denen sie wissen müssen, dass so ein Preis nur durch illegal Beschäftigte zustande kommen kann", sagt Heerdt: "Damit machen sie sich zum Hehler."

Negation. Das FVG kommt im Korpus 18x negiert vor. In allen Fällen wird die Negation durch ein unmittelbar vor der Präposition zu stehendes nicht (bzw. 1x nicht mehr und 1x nie) ausgedrückt:

(33) Kommt diese Mehrheit in der Beratung über die Schuldfrage nicht zustande, ist freizusprechen.

(34) Der Handel kommt nicht zustande.

Zur Komplementierung. Das FVG zustande kommen ist "gesättigt" und lässt keine Komplementierung zu.

Subjekt. An der Subjektstelle dominiert unter semantischem Gesichtspunkt eindeutig die Kategorie [+Abstr] (61x) vor [+Konkr] (6x) und [+Koll] $(4 \mathrm{x})$.

Unter den äußerst vielseitigen Abstrakta können folgende mehr oder weniger einheitliche Gruppen als wichtigste, das Ende eines längeren Entstehungsprozesses markierende Bedeutungsfelder herausdestilliert werden:

- 'Reform': 9 Belege (4x Reform; je 1x Gesetz, Finanzierungskonzept, Pflegeversicherung, Normen und Steuerbefreiung) [vgl. 27],

- 'Entscheidung': 9 Belege (2x Entscheidung; je 1x Aussage, Absichtserklärung, Urteil, Vereinbarung, Einstellung, Position und Boykottbewegung) [vgl. 28],

- 'Geschäft': 8 Belege (je 2x Geschäft und Handel; je 1x Deal, Beteiligung, Investition und Zusammenschluss) [vgl. 29; 34],

- '(politische) Mehrheit': 7 Belege (6x Mehrheit, 1x Koalition) [vgl. 30; 33],

- 'Treffen': 7 Belege (je 1x Treffen, Gespräch, Kontakte, Vierer-Konferenz, Dayton, Verbindungen und Verhältnis) [vgl. 31],

- 'Preis': 4 Belege (2x Preis; je 1x Ausgabe und Summe) [vgl. 32].

Die 6 Konkreta drücken entweder ein 'Bauwerk' wie in [35] oder das 'Erzeugnis eines künstlerischen bzw. kreativen Prozesses' (Buch, Gemälde, Film) wie in [36] aus:

(35) So kamen unterirdische Verbindungsräume zwischen Alt- und Neubau zustande, die, wegen der Tiefgarage darunter, als flacher Pyramidenstumpf nach oben drängen. 
(36) Dass der Film in dieser Form zustande gekommen ist, ist maßgeblich das Verdienst eines überaus professionell arbeitenden Teams, des Kameramanns und der trotz Frust am Set disziplinierten Schauspieler.

Die dritte vertretene Kategorie ist diejenige der Kollektivbegriffe mit nur 3 Belegen [vgl. 37; 38]:

(37) So viele Soldaten fielen durch, dass die Spezialeinheit nicht zustande kam.

(38) Ohne klammheimlich gewobene Absprachen zwischen den starken Männern der einzelnen Parteien wäre die Zwei-Kammern-Kommission wohl gar nicht zustande gekommen.

\section{III. zur/auf die Welt kommen}

(geboren werden)

Für das FVG zur/auf die Welt kommen weist das Spiegel-Korpus 62 Belege auf. Dabei kommt es 48mal in der Variante mit der kontrahierten Präposition zur, 12mal mit der gleichen Bedeutung in der Variante auf die und 2mal in der seltenen Variante mit übertragener Bedeutung in die vor. Typische Belege sehen wie folgt aus:

(39) Der Sohn, der im Winter darauf zur Welt kommt, soll - wie anders? - Jesus heißen.

(40) Verzweifelte Eltern überlassen immer häufiger ihre Kinder, besonders wenn diese mit Behinderungen zur Welt kommen, sich selbst oder der Fürsorge des Staats.

(41) "Die Zukunft riecht nach Juchten, nach Blut, nach Gottlosigkeit und nach sehr vielen Prügeln. Ich rathe unsern Enkeln, mit einer sehr dicken Rückenhaut zur Welt zu kommen."

(42) Primor, 1935 in Tel Aviv zur Welt gekommen, ist Sohn einer Deutschen und eines Holländers.

(43) Wir kommen nun mal nicht alle mit den gleichen inneren Entwicklungsmöglichkeiten auf die Welt.

(44) Das Kind kam als praller Neunpfünder auf die Welt - aber etwas stimmte nicht.

Zur Erweiterbarkeit. Das Syntagma wird im Normalfall nicht erweitert. In einem einzigen Beleg weist die PG eine ungewöhnliche Erweiterung durch ein Adjektiv auf, das sprachspielerisch zur Unterscheidung zwischen dem Kult um den Maler Keith Haring in der virtuellen Welt des Internet und dessen reellen Lebensdaten dient:

(45) Zur wirklichen Welt kam er [Keith Haring] 1958 in der Kleinstadt Kutztown in Pennsylvania, und ein wenig blieb er immer der Junge vom Land, auch wenn er sich rasch in die moderne Zeit einklickte.

Zur Komplementierung. Das FVG zur/auf die Welt kommen eröffnet keinen Platz für Komplemente irgendwelcher Art.

Subjekt. In der Verteilung der Subjekte nach semantischen Kriterien überwiegen eindeutig die [+Hum] [52 Belege, vgl. 39-44]. Doch daneben treten auch [+Anim] (5 Belege) und - im übertragenen Sinne - [+Konkr] (3 Belege) und [+Abstr] (2 Belege) auf.

Unter den "menschlichen Wesen" verlagern sich die Gewichte im Vergleich zu den Akkusativobjekten beim Syntagma zur/auf die Welt bringen deutlich. Unter den einzelnen 
Substantiven dominieren zwar weiterhin Kind [10x, vgl. 40; 44] sowie Sohn, Tochter und Junge (je 2x), doch den Großteil der Subjekte liefern hier Personalpronomen und Personennamen von meist erwachsenen oder sogar bereits verstorbenen Menschen [vgl. 42; 45], deren Lebenslauf im Artikel geschildert wird (insg. 30 Belege).

In 5 Belegen wird von der Geburt anderer Lebewesen berichtet. Dabei handelt es sich in 3 Fällen um genmanipulierte oder geklonte Tiere wie in [47; 48]:

(46) Die Jungtiere kommen häufig in Höhlen zur Welt, aus denen zuvor andere Tiere, etwa Warzenschweine, vertrieben wurden.

(47) Nach der üblichen Tragzeit kommt [das Schaf] Dolly zur Welt - genetisch mit ihrer Genmutter identisch.

(48) Schon im letzten August seien die beiden Rhesusäffchen zur Welt gekommen, verkündete er geklont aus dem Gewebe anderer, kurz zuvor gezeugter Embryonen.

In 3 Belegen steht ein Konkretum an der Subjektstelle. Beiden Belegen ist die spontane Entstehung eines Gegenstands gemeinsam. Während es in [49] um die plötzliche Entstehung von Teilchen und damit des Lebens durch den Urknall geht, beschreibt [50] die Geburt einer neuen Stadt, die am Reißbrett entstand und von A bis Z nach sozialistischen Maßstäben eingerichtet wurde:

(49) Nach der Vorstellung von Peter Higgs nämlich kamen im Urknall alle Teilchen gewichtslos zur Welt.

(50) Sie waren das Paillettenkleid einer Stadt, die im Blaumann auf die Welt kam: Eisenhüttenstadt - "die erste sozialistische Stadt auf deutschem Boden".

Schließlich weist das Korpus im Zusammenhang mit dem Syntagma in die Welt kommen 2 Belege mit einem Abstraktum an der Subjektstelle auf:

(51) Der Titel: "Wie kommt das Neue in die Welt?"

(52) So kam der Mythos vom Wirtschaftswunder in die Welt.

IV. (für jn/etw.) in Frage kommen

(geeignet sein; in Betracht kommen)

Im FVG (für jn/etw.) in Frage kommen (61 Belege) kommt die Präposition in immer in Verbindung mit dem Nullartikel vor. Die PG wird nie erweitert, wie in folgenden Belegen:

(53) Und dafür kommt nur einer in Frage.

(54) Vickie konnte dafür in Frage kommen.

(55) Natürlich, so schränkt Thierse ein, müsse man sehen, ob für den Job auch ein original Ostdeutscher in Frage komme.

Negation. Das Besondere an diesem FVG ist, dass es im Korpus nicht weniger als 41mal, d.h. in über zwei Dritteln aller Belege, negiert vorkommt. Die Negation wird in fast allen Fällen durch ein unmittelbar vor der PG in Frage stehendes nicht (bzw. 4x nicht mehr, 2x auf keinen Fall und je 1x nocht nicht und nie) ausgedrückt: 
(56) Damals waren sich eigentlich alle einig, dass eine weitere Verlängerung nicht in Frage kommt.

(57) Die normalen Verfahren von Pressen, Ziehen, Walzen, mit denen metallische Leiter üblicherweise in Form gebracht werden, kommen deshalb nicht in Frage.

(58) Aber mit meinem Charaktergesicht kamen viele Rollen einfach nicht in Frage.

(59) Ein Rückzieher kam nicht mehr in Frage, schon allein, weil Naganos Stadtväter nicht auf die teuren Infrastruktur-Projekte verzichten wollten.

In lediglich 2 Belegen steht das Negationswort nicht direkt vor der PG, sondern vor einem durch für eingeleiteten Präpositionalobjekt:

(60) Dass Meryl Streep oder Meg Ryan nicht für die Smilla-Rolle in Frage gekommen wären, ist ja klar, doch Julia Ormond finde ich optimal.

(61) Meine Karriere - mal sehen; vorläufig werden Parteigänger wie ich noch nicht für das Amt des Chefs der Sonderverwaltungszone in Frage kommen.

Diese Stellung von nicht als Satznegation ist zwar insgesamt sicher weniger geläufig, aber zweifellos auch möglich (Sie kommen dafür nicht in Frage/Sie kommen nicht dafür in Frage). Jedenfalls ergibt sich aus dem Ko(n)text keinerlei Hinweis dafür, dass es sich in diesen beiden Fällen um eine Sondernegation (Teilnegation) handeln würde im Sinne von: Sie kommen nicht für die Smilla-Rolle, sondern vielleicht für eine andere in Frage.

Zur Komplementierung. Das Syntagma lässt ein fakultatives, durch für eingeleitetes Präpositionalobjekt zu (19 Belege).

Präpositionalobjekt. 19 Belege enthalten ein durch für eingeleitetes Präpositionalobjekt. Enthält dieses Präpositionalobjekt ein Abstraktum, dann drückt es (im Zusammenhang mit einem menschlichen Subjekt) ein anvisiertes Ziel oder im allgemeinen etwas Erstrebenswertes aus [11x, vgl. 53-55; 60-63]. Enthält es ein Nomen der Kategorie [+Hum], dann drückt es eine anvisierte Zielperson aus [8x, vgl. 64-65].

(62) Und für eine weitere Studie mit einem der neuen Proteasehemmer kam sie nicht in Frage, weil sie zu lange AZT genommen hatte.

(63) Kann man den Belgiern, die für den ersten Schub nicht in Frage kommen, zumuten, im eigenen Land am Sitz der Kommission mit dem Euro anstatt dem gewohnten belgischen Franc zu bezahlen?

(64) Es ist die einzige Form des Wohnens, die für mich in Frage kommt.

(65) Für jemanden, der die heilige Maria noch mehr verehrt als jungfräuliche Mädchen die Back Street Boys, kommt folglich gar keine andere Automarke in Frage.

Adverbial. In 9 Belegen tritt zwar ebenfalls ein Satzglied mit "für + NG" auf (wobei das Nomen immer ein Vertreter der Kategorie [+Hum] ist). Doch handelt es sich dabei nicht um die für dieses FVG spezifische Ergänzung [semantisch: 'anvisiertes Ziel' oder 'betroffene Person(engruppe)'], sondern um ein - grundsätzlich in jeden Satz einfügbares - Adverbial [semantisch: 'Nennung der Person(engruppe), welche die Aussage beurteilt']. Dass es sich dabei um zwei verschiedene syntaktische Positionen handelt, geht auch daraus hervor, dass sie kumulierbar sind: Für mich kommt diese Rolle für mich/ihn... nicht in Frage. 
(66) Ein Kompromiß nur für die Rente kommt für den CDU-Unterhändler allerdings nicht in Frage: "Entweder wir lösen Rente und Steuer im Paket, oder es geht gar nichts."

(67) Als Instrument zur Bekämpfung der organisierten Kriminalität kommt eine staatlich kontrollierte Versorgung der Süchtigen für ihn nicht in Frage.

Die semantische Funktion der Präpositionalgruppen in den beiden obigen Belegen lässt sich eindeutig definieren. Der CDU-Unterhändler ist keineswegs als Zielperson der Rente zu verstehen, der Polizeipräsident noch weniger als Zielperson der staatlich kontrollierten Drogenabgabe. Vielmehr liefert das Adverbial in [66] wie in [67] die Identität der Person, deren Meinung reflektiert wird:

(66') Ein Kompromiss kommt nach der Meinung/in den Augen /nach der Beurteilung des CDUUnterhändlers nicht in Frage.

(67') Seiner Meinung nach/In seinen Augen/Nach seiner Beurteilung kommt die staatlich kontrollierte Versorgung der Süchtigen nicht in Frage.

In 4 Belegen schließlich tritt ein durch die Satzteilkonjunktion als eingeleitetes Satzglied auf, durch das semantisch betrachtet dem Subjekt eine bestimmte Rolle zugeordnet wird:

(68) Wenn Sie als Täterin ausscheiden, kommt eigentlich nur Reinhard Weimar als Mörder der Mädchen in Frage.

(69) Das Government House kommt für Tung als Regierungssitz nicht in Frage - "ich habe gehört, das Feng Shui dort ist schlecht", sagt er.

Subjekt. An der Subjektstelle dominieren unter semantischem Gesichtspunkt zwar eindeutig die Kategorien [+Hum] (19 Belege) und vor allem [+Abstr] (35 Belege), doch andere Kategorien sind dabei keineswegs ausgeschlossen: 6mal tritt ein Konkretum als Subjekt auf [vgl. 69], in einem Beleg ein Kollektivbegriff.

\subsection{Vergleich des Spiegel-Korpus mit den Kodifizierungen in den Wörterbüchern}

Interessant ist die Frage, wieviele und welche der 10 häufigsten FVG des Spiegel-Korpus mit dem Verb kommen in vier gängigen deutschsprachigen Wörterbüchern ${ }^{8}$ vorkommen. Die Suche erfolgte zunächst unter dem Lemma "kommen", erst in einem zweiten Schritt unter dem jeweiligen Substantiv. Das Ergebnis sieht folgendermaßen aus:

\footnotetext{
8 (1) Duden (1999): Das große Wörterbuch der deutschen Sprache in 10 Bänden; (2) Duden (1996): Deutsches Universalwörterbuch; (3) Götz et al. (31994): Langenscheidts Großwörterbuch Deutsch als Fremdsprache; (4) Wahrig (2000): Deutsches Wörterbuch.
} 


\begin{tabular}{|c|c|c|c|c|}
\hline & Duden 10 & $\begin{array}{c}\text { Duden } \\
\text { Universal }\end{array}$ & $\begin{array}{c}\text { Langenscheidt } \\
\text { DaF }\end{array}$ & Wahrig \\
\hline 1. ums Leben kommen & $X$ & $X$ & $\mathrm{O}$ & $\mathrm{X}$ \\
\hline 2. zustande $\sim$ & $\mathrm{O}$ & $\mathrm{O}$ & $\mathrm{O}$ & $\mathrm{O}$ \\
\hline 3. zur/auf die Welt & $\mathrm{O}$ & $\mathrm{O}$ & $\mathrm{O}$ & $X$ \\
\hline 4. in Frage & $\mathrm{O}$ & $\mathrm{o}$ & $\mathrm{O}$ & $\mathrm{O}$ \\
\hline 5. auf eine Idee $\sim$ & - & - & - & - \\
\hline 6. zu einem Ergebnis & $\mathrm{O}$ & - & $X$ & $\mathrm{O}$ \\
\hline 7. auf den Markt & $\mathrm{O}$ & - & - & - \\
\hline 8. zugute $\sim$ & $\mathrm{O}$ & $\mathrm{O}$ & $\mathrm{O}$ & $\mathrm{O}$ \\
\hline $\begin{array}{l}\text { 9. abhanden } \sim \\
\text { in js Hände }\end{array}$ & $\begin{array}{l}\mathrm{O} \\
\mathrm{O}\end{array}$ & $\begin{array}{l}\mathrm{O} \\
\mathrm{O}\end{array}$ & $\begin{array}{l}0 \\
- \\
\end{array}$ & $\begin{array}{l}\mathrm{O} \\
\mathrm{X} \\
\end{array}$ \\
\hline 10. zu einem Schluss $\sim$ & $\mathrm{o}$ & $\mathrm{o}$ & $\mathrm{O}$ & - \\
\hline
\end{tabular}

Tab. 3: Die 10 häufigsten FVG mit kommen in vier gängigen Wörterbüchern ( $\mathrm{X}=$ gebucht unter "kommen"; o = gebucht unter dem jeweiligen Substantiv; - = nicht gebucht)

Unter dem Lemma "kommen" wird kein einziges der Syntagmen aus unserer Top-Ten-Liste in allen Wörterbüchern aufgeführt. Als einziges wird die Nummer (1) ums Leben kommen in 3 Werken verzeichnet.

Beim FV kommen erweist sich Wahrig als am vollständigsten. Dabei bleibt das Ergebnis mit insgesamt 3 Syntagmen allerdings bescheiden: Wahrig nennt neben der Nummer (1) lediglich auf die Welt kommen (Nr. 3) und in einem Beispielsatz in js Hände kommen (Nr. 8B). In beiden Duden-Wörterbüchern steht ein Syntagma zum Schluss kommen, das inhaltlich aber nicht dem auf Rang 10 stehenden Syntagma zu einem <bestimmten> Schluss kommen (mit der Unterbedeutung 'Konklusion, Folgerung') entspricht, sondern die im Korpus bloß ein einziges Mal belegte, "banalere" Bedeutung 'Ende' enthält. Langenscheidt DaF schließlich führt als einziges Syntagma aus der Top-Ten-Liste zu einem <bestimmten $>$ Ergebnis kommen (Nr. 6) auf.

Die Suche unter dem jeweiligen Substantiv bringt glücklicherweise eine klare Korrektur: Im zehnbändigen Duden-Wörterbuch fehlt nach diesem zweiten Schritt nur noch ein, in den anderen Wörterbüchern 3 Syntagmen.

Als einziges Syntagma wird auf <eine bestimmte> Idee kommen (Nr. 5) von keinem der Wörterbücher aufgeführt. In den beiden Duden-Werken steht dagegen das zweiwertige Syntagma jn auf <eine bestimmte> Idee bringen im Beispielsatz er [bzw. sie] hat mich erst auf diese Idee gebracht.

Das FVG auf den Markt kommen (Nr. 7) seinerseits kommt nur im zehnbändigen Duden im Beispielsatz ein neues Produkt ist auf den Markt gekommen vor. Die PG auf den Markt wird sowohl von Langenscheidt DaF (etw. auf den Markt bringen/werfen) als auch von Wahrig (einen neuen Artikel auf den Markt bringen) ausschließlich im Zusammenhang mit zweiwertigen Syntagmen aufgeführt; im Universalwörterbuch kommt sie gar nicht vor.

In Bezug auf das FVG auf die/zur Welt kommen (Nr. 3) erweist sich Langenscheidt DaF als übersichtlicher als seine Konkurrenten. Hier wird das Syntagma nämlich an derselben Stelle 
gleich mit beiden Präpositionen aufgeführt. In den Duden-Wörterbüchern kommen zwar auch beide Varianten vor, doch sie liegen recht weit auseinander.

Obwohl das Universalwörterbuch die Syntagmen ein $<$ bestimmtes $>$ Ergebnis bringen und $z u$ einem < bestimmten> Ergebnis führen nennt, führt es als einziges das einwertige FVG $z u$ einem < bestimmten $>$ Ergebnis kommen (Nr. 6) nicht auf.

Auch ein Blick in Engel/Schumacher (1978) lohnt sich. Von den Top-Ten aus dem SpiegelKorpus kommen nur gerade die Nummern (3) zur Welt kommen (ohne die seltenere Variante auf die Welt kommen) und (4) in Frage kommen vor. Ein besonderes Augenmerk verdient dagegen der prägnante Beispielsatz zu (4), Er kommt für diese Arbeit nicht in Frage. Dieser enthält - geradezu prototypisch - neben einem menschlichen Subjekt ein durch für eingeleitetes Präpositionalobjekt mit Abstraktum, welches das vom Agens anvisierte Ziel ausdrückt.

\subsection{Vergleich des Spiegel-Korpus mit der Darstellung in Grammatiken}

Es soll an dieser Stelle untersucht werden, welche der 10 häufigsten FVG des Spiegel-Korpus mit dem Verb kommen in den drei DaF-Grammatiken von Helbig/Buscha (2001), Bresson (1988) und Heringer (1997) vorkommen und welche zusätzlichen Syntagmen - eventuell darin aufgeführt werden.

Für das Funktionsverb kommen führen Helbig/Buscha (2001: 78) nicht weniger als 18 Beispiele auf. Davon gehört lediglich in Frage kommen (Rang 4) zu den Top-Ten in unserem Korpus (vgl. Tab. 2).

In dieser Grammatik kommen außerdem folgende FVG vor, die im Spiegel-Korpus jedoch einen sehr unterschiedlichen Häufigkeitsgrad aufweisen: Neben den frequenten in Gang kommen (32 Belege); zur Ruhe kommen (23) und zum Vorschein kommen (16) finden sich zwar relativ häufige Syntagmen wie zum Ausdruck kommen; zum Stillstand kommen (je 7 Belege) und in Fahrt kommen; in Schwung kommen (je 6 Belege), aber auch solche, die nur eine geringe Anzahl von Belegen aufweisen: zum Abschluß kommen; zum Ausbruch kommen (je 3 Belege), zur Kenntnis kommen (2) und in Bewegung kommen; zur Verhandlung kommen; zur Vernunft kommen (je ein Beleg).

Schließlich finden sich 4 Syntagmen, die im Spiegel-Korpus nicht belegt sind: zu Ansehen kommen; in/zur Anwendung kommen; zur Blüte kommen und zur Versteigerung kommen.

Bresson (1988) nennt bloß ein Syntagma mit dem Funktionsverb kommen, in Verlegenheit kommen, für welches das Spiegel-Korpus einen einzigen Beleg aufweist. Die Wahl dieses Syntagmas lässt sich wohl vor allem dadurch erklären, dass Bresson dessen Pendant in Verlegenheit bringen aufführt.

Heringer (1997) schließlich führt 9 Beispiele auf. Darunter ist das bereits bei Helbig/Buscha aufgeführte in Gang kommen mit 32 Belegen das häufigste (Rang 12). Es folgen zu Hilfe kommen (12 Belege); zum Ausdruck kommen (7); zur Entscheidung kommen (2) und ins Schwitzen kommen (ein Beleg). 4 Beispiele schließlich kommen in unserem Korpus gar nicht 
vor: in die Debatte kommen; zum Kochen kommen; ins Rollen kommen und - auch dieses bereits bei Helbig/Buscha vorhanden - zur Anwendung kommen .

Auffallend ist in dieser Liste, dass von 9 Beispielen 3 einen substantivierten Infinitiv enthalten (Schwitzen; Kochen; Rollen). Doch obwohl diese Bildungsmöglichkeit theoretisch und gerade im Sinne des bereits erwähnten Prinzips der Reihenbildung von FVG - durchaus interessant ist, erfreut sie sich in unserem Korpus nur einer sehr mäßigen Beliebtheit (die häufigsten sind mit je 3 Belegen Plaudern; Rutschen; Stocken und Tragen).

Fazit: Als einzige der 3 berücksichtigten Grammatiken führt diejenige von Helbig/Buscha ein Syntagma aus unseren Top-Ten auf, ohne jedoch die allerhäufigsten zu nennen. Dies ist umso erstaunlicher, als sie unter dem Funktionsverb bringen das Syntagma ums Leben bringen aufführt. Von daher gesehen hätte man die Nummer (1) ums Leben kommen erwarten können.

Etwas überraschend ist an dieser Stelle außerdem die Anzahl von Beispielen, die in unserem Korpus nur einmal (5) oder gar nicht belegt sind (7) sowie die Tatsache, dass eines davon (zur Anwendung kommen) gleich in zwei Werken vorkommt.

\section{$6 \quad$ Vergleich Spiegel/Tages-Anzeiger (COSMAS II)}

Solche Ergebnisse, wie sie hier vorliegen, sind natürlich korpus- und gattungsabhängig. Außerdem stammen sie aus einem verhältnismäßig bescheidenen Korpus.

Um diesen möglicherweise eine breitere Gültigkeit zu verleihen oder um sie zu widerlegen, habe ich beschlossen, sie in einem weiteren Kontext, im Rahmen von COSMAS II, zu überprüfen. Berücksichtigt wurde das Tages-Anzeiger-Korpus, d.h. alle Ausgaben der Zürcher Tageszeitung in den 5 Jahren zwischen 1996 und 2000 mit insgesamt genau 103'315 Belegen für das Verb kommen (zur Erinnerung: 6'441 beim Spiegel-Korpus).

Dem Test unterzogen wurden die 25 häufigsten Syntagmen mit kommen aus dem SpiegelKorpus. Das Ergebnis sieht folgendermaßen aus: 


\begin{tabular}{|c|c|c|c|c|c|}
\hline Rang & FVG & $\begin{array}{l}\text { Spiegel- } \\
\text { Korpus }\end{array}$ & Rang & FVG & $\begin{array}{r}T A- \\
\text { Korpus } \\
\end{array}$ \\
\hline 1. & ums Leben kommen & 79 & 1. & (für jn/etw.) in Frage kommen & $2 ' 099$ \\
\hline 2. & $\begin{array}{l}\text { zustande kommen } \\
\text { zu Stande kommen }\end{array}$ & $\begin{array}{r}71 \\
-\end{array}$ & 2. & $\begin{array}{l}\text { zustande kommen } \\
\text { zu Stande kommen }\end{array}$ & $\begin{array}{r}1 \\
6433 \\
637\end{array}$ \\
\hline 3. & $\begin{array}{l}\text { zur Welt kommen } \\
\text { auf die Welt kommen } \\
\text { in die Welt kommen }\end{array}$ & $\begin{array}{rr}48 & 62 \\
12 & \\
2 & \\
\end{array}$ & 3. & ums Leben kommen & 1 '869 \\
\hline 4. & (für jn/etw.) in Frage kommen & 61 & 4. & zu einem <bestimmten $>$ Schluss kommen & $1^{\prime} 706$ \\
\hline 5. & auf $<$ eine bestimmte> Idee kommen & 60 & 5. & $\begin{array}{l}\text { jm zugute kommen } \\
\text { jm zu Gute kommen }\end{array}$ & $\begin{array}{r}1 \\
1 \\
13 \\
\end{array}$ \\
\hline 6.7 & zu einem <bestimmten $>$ Ergebnis kommen & 59 & 6. & zum Einsatz kommen & $1^{\prime} 048$ \\
\hline 7. & auf den Markt kommen & & 7. & auf den Markt kommen & 710 \\
\hline 8. & $\begin{array}{l}\text { im zugute kommen } \\
\text { jm zu Gute kommen }\end{array}$ & & 8. & zum Zug(e) kommen & 524 \\
\hline 9. - & $\begin{array}{l}\text { (jm) a handen kommen } \\
\text { in } \mathrm{js} /<\text { beste }>\text { Hände }\end{array}$ & & 9. & $\begin{array}{l}\text { zur Welt kommen } \\
\text { auf die Welt kommen } \\
\text { in die Welt kommen }\end{array}$ & $\begin{array}{r}361 \\
139 \\
- \\
\end{array}$ \\
\hline 10. & zu einem <bestinmteri $>$ Schluss kommen & 43 & 10. & auf <eine bestimmte> Idee kommen & 443 \\
\hline 11. & an die Macht kgnmen & 34 & 11. & $\begin{array}{l}\text { (jm) abhanden kommen } \\
\text { in js/<bestimmte> Hände kommen }\end{array}$ & $\begin{array}{r}390 \\
37 \\
\end{array}$ \\
\hline 12. & in Gang kommen & 32 & 12. & zu Wort kommen & 365 \\
\hline 13. & zu Wort kommen & 29 & 13. & jm in den Sinn kommen & 354 \\
\hline $14 .^{7}$ & zum Einsatz kommgn & 28 & 14. & zum Vorschein kommen & 354 \\
\hline 15. & ans Licht kompzen & 27 & 15. & jm/etw. auf die Spur kommen & 232 \\
\hline 16. & jm/etw. auf/die Spur kommen & 27 & 16. & in Gang kommen & 211 \\
\hline 17. & $\begin{array}{l}\text { zurecb/ kommen } \\
\text { zy Recht kommen }\end{array}$ & 27 & 17. & $\begin{array}{l}\text { zurecht kommen } \\
\text { zu Recht kommen }\end{array}$ & $\begin{array}{r}207 \\
1 \\
\end{array}$ \\
\hline 18. & zur Ruhe kommen & 23 & 18. & zur Ruhe kommen & 204 \\
\hline 19.7 & zum Zug(e) kommen & 23 & 19. & jm in die Quere kommen & 187 \\
\hline 20. & (mit jm) ins Geschäft kommen & $2 x$ & 20. & an die Macht kommen & 185 \\
\hline 21. & jm in den Sinn kommen & 20 & 21. & (mit jm) ins Gespräch kommen & 181 \\
\hline 22. & vor Gericht kommen & 17 & 22. & zu einem <bestimmten $>$ Ergebnis kommen & 160 \\
\hline 23. & (mit jm) ins Gespräch kommen & 17 & 23. & ans Licht kommen & 125 \\
\hline 24. & jm in die Quere kommen & 16 & 24. & vor Gericht kommen & 118 \\
\hline 25. & zum Vorschein kommen & 16 & 25. & (mit jm) ins Geschäft kommen & 81 \\
\hline
\end{tabular}

Tab. 4: Vergleich: die 25 häufigsten FVG im Spiegel-Korpus im TA-Korpus

Wie man sieht, überschneiden sich die Top-Ten im TA-Korpus fast gänzlich mit der SpiegelRangfolge. Auch der Frequenzunterschied zwischen zur Welt kommen und auf die Welt kommen wird bestätigt.

Die einzigen Ausnahmen bilden unsere Nummern (6) (zu einem <bestimmten> Ergebnis kommen), das im TA-Korpus auf Rang 22 abrutscht, und (9), das im TA-Korpus nur wenige Plätze dahinter liegt (Rang 11).

Der beträchtliche Unterschied bei zu einem <bestimmten> Ergebnis kommen ließe sich vielleicht durch die Provenienz der beiden Korpora erklären: Das Syntagma ist wohl in der deutschsprachigen Schweiz allgemein weniger frequent als in Norddeutschland. Ein Hinweis darauf ist, dass das bedeutungsähnliche zu einem <bestimmten> Schluss kommen im TAKorpus weit oben liegt (Rang 4 gegenüber Rang 10 im Spiegel-Korpus).

Das häufige Vorkommen von zum Einsatz kommen und wohl auch - wenn auch in geringerem Maße - von zum Zug(e) kommen im TA-Korpus lässt sich dagegen durch die - im Vergleich zum Spiegel-Korpus - hohe Anzahl von Sportberichten erklären. 
Interessant ist weiter, dass unter den Top 25 4mal der genau gleiche Rang vorliegt: 2 (zustande kommen), 7 (auf den Markt kommen), 17 (zurecht kommen), 18 (zur Ruhe kommen).

Der größte Nutzen einer solchen Überprüfung aber ist wohl, dass der Tages-Anzeiger zwischen den Rängen 1 bis 5 größere Differenzen bietet, während diese beim Spiegel recht klein und damit in einem gewissen Sinn "zufällig" sind (zwischen 79 und 60 Belege). Dass im TA-Korpus nicht mehr ums Leben kommen dominiert, sondern in Frage kommen, entspricht wohl eher der Intuition eines sprachsensiblen Muttersprachlers.

Natürlich könnte es sein, dass bei der Wahl des TA-Korpus als Leitkorpus noch andere FVG auftauchen. Rein theoretisch wäre es möglich, dass im TA-Korpus ein FVG, das ich gar nicht erfasst habe, die erste Stelle einnähme. Aber dies scheint doch eher unwahrscheinlich. Möglich wäre auch, dass eine Gesamtuntersuchung des Tages-Anzeiger wohl einige FVG unter die ersten 25 spülen würde, die beim Spiegel weiter unten liegen oder gar nicht vorkommen. Diesen Beweis werde ich selbst aber nicht erbringen: In diesem Fall überlasse ich die Arbeit gern anderen: Es sind ja "nur" etwas über 100'000 Belege, die einzeln unter die Lupe genommen werden müssten...

\section{Literaturangaben}

Quellen:

Der Spiegel. CD-Rom Ausgabe 1997. Hamburg: Spiegel-Verlag 1998.

COSMAS II: http://www.ids-mannheim.de/cosmas2/

\section{Literatur:}

Bresson, Daniel (1988): Grammaire d'usage de l'allemand contemporain. Paris.

Daniels, Karlheinz (1963): Substantivierungstendenzen in der deutschen Gegenwartssprache. Nominaler Ausbau des verbalen Denkkreises. Düsseldorf.

Duden (1996): Deutsches Universalwörterbuch [A-Z]: auf der Grundlage der neuen amtlichen Rechtschreibregeln. Bearb. von Günther Drosdowski und der Dudenredaktion. 3., neu bearb. und erw. Aufl. Mannheim/Zürich etc.

Duden (1999): Das große Wörterbuch der deutschen Sprache in 10 Bänden. Hrsg. vom Wissenschaftlichen Rat der Dudenredaktion. Mannheim.

Engel, Ulrich/Schumacher, Helmut (1978): Kleines Valenzlexikon deutscher Verben. 2., durchges. Auflage. Tübingen.

Götz, Dieter et al. (eds.) (31994): Langenscheidts Grosswörterbuch Deutsch als Fremdsprache. Das neue einsprachige Wörterbuch für Deutschlernende. Berlin.

Haß-Zumkehr, Ulrike (2000): "Wortschatz ist mehr als 'viele Wörter'. Die Aufgaben der Abteilung Lexik des IDS". Sprachreport 2/2000: 2-7.

Helbig, Gerhard/Buscha, Joachim (2001): Deutsche Grammatik. Ein Handbuch für den Ausländerunterricht. Berlin/München.

Hentschel, Elke/Weydt, Harald (1990): Handbuch der deutschen Grammatik. Berlin/New York.

Heringer, Hans Jürgen (1997): Grammaire allemande. Paris. 
Hofstetter, Anne (1989): Funktionsverbgefüge. Abgrenzungsproblematik - Deutschfranzösisches Lexikon. Neuchâtel: unveröffentlichte Diplomarbeit zur Erlangung der Licence ès lettres.

Kamber, Alain/Näf, Anton (2001): "'Locutions verbales' en français - 'Funktionsverbgefüge' en allemand: essai de bilan descriptif et terminologique". In: Colombat, Bernard/Savelli, Marie (eds.): Métalangage et terminologie linguistique. Actes du colloque international de Grenoble. Leuven: 901-913.

Kamber, Alain (im Druck): Funktionsverbgefüge - empirisch. Eine korpusbasierte Untersuchung in fremdsprachendidaktischer Perspektive. Dissertation zur Erlangung der Doktorwürde an der Philosophischen Fakultät der Universität Neuchâtel.

Polenz, Peter von (1963): Funktionsverben im heutigen Deutsch. Sprache in der rationalisierten Welt. Düsseldorf.

Pottelberge, Jeroen van (2001): Verbonominale Konstruktionen, Funktionsverbgefüge. Heidelberg.

Sinclair, John (1986): "First Throw Away Your Evidence". In: Leitner, Gerhard (ed.): The English Reference Grammar. Language an Linguistics, Writers and Readers. Tübingen: 56-64. (= Linguistische Arbeiten 172).

So, Man-Seob (1991): Die deutschen Funktionsverbgefüge in ihrer Entwicklung vom 17. Jahrhundert bis zur Gegenwart. Eine sprachhistorische Untersuchung anhand von populärwissenschaftlichen Texten. Trier.

Vivès, Robert (1983): Avoir, prendre, perdre: constructions à verbe support et extensions aspectuelles. Paris.

Wahrig, Gerhard (2000): Deutsches Wörterbuch. Gütersloh. 